\title{
UNDERSTANDING GENETIC ALGORITHMS IN ARCHITECTURE
}

\author{
Mohammad Latifi \\ Department Of Art And Architecture, Tehran Science And Research Branch, Islamic Azad \\ University, Tehran, Iran \\ Mohammadjavad Mahdavinejad \\ Department Of Architecture, Tarbiat Modares University, Tehran, Iran \\ Darab Diba \\ Department Of Architecture And Urban Planning, Central Tehran Branch, Islamic Azad University, \\ Tehran, Iran
}

\begin{abstract}
Genetic algorithms (GAs) are computational techniques based upon evolution. They have been recently introduced in architecture to study present complexities in form and function. Despite growing trends and inclinations to use GAs, there is not an organized study and instruction about its usage in architecture.

This library research, while examining the structure of GAs, seeks answers to these questions:

1. What is the necessity of GAs in architecture today?

2. Are GAs used to meet special needs of architecture or are they merely complex forms?

3. Haw can GAs come to our assistance in design? And last but not least 4. Does GAs serve reality or unreal abstract forms of an imaginative world!?

To answer these questions, the present research studies GAs in architecture and other disciplines. It also investigates their functions in architecture and finally explains introductory parametric operations in this field.
\end{abstract}

Keywords: Genetic Algorithm, Genetic Operators, Parametric Architecture, Optimization

\section{INTRODUCTION}

Since the 1990s, there was a change in approach leading architects, so that, evolutionary biology technologies have been used to investigate or to depict the complexities of modern architecture. Since the amount of information and level of complexity in most construction projects, are larger than the ability of designers to understand and predict them in a way that traditional methods of design, we can not solve, this complexity at the right time in the midst other evolutionary and dynamic techniques, genetic algorithms, have been used, as a means of optimizing the architecture.

Genetic algorithm used in the first step in resolving structural problems, such as structural and facility structure, and in the next step, it is used as the probability of random solutions to solve optimization problems and research issues. Genetic algorithms are used as new and unexpected conceptual areas in order to create a symbol and innovative solutions in the form of processes, new structures can be created with a lot of complexity to it.

\section{GENETIC ALGORITHMS}

GA, inspired Darwin's theory of evolution and genetics, and it is based on superior survival or natural selection. A common use of genetic algorithms is used as a function optimization. Also, genetic algorithms are useful tools in pattern recognition, feature selection, image understanding, optimization, evolution, automated programming, machine learning, and teaching behavior of the robot and so on. [1]

Although the work was done by a biologist named Fraser, in the field of modeling evolution in biological systems in the late ' $60 \mathrm{~s}$, but genetic algorithms, for engineering applications, and for today, was first proposed by John Henry Holland, scientist Computer University of Michigan in 1975. His 
work is the beginning of all efforts to use genetic algorithms in engineering. After that, the work of Kenneth A De Jong, in 1975, provided the intellectual foundation for discussion, evaluate and compare several genetic algorithms. [2] The algorithm, inspired by nature is based on the principle of Survival of the fittest. [3] Although genetic algorithm was proposed after the algorithm evolutionary strategy, but it is, the most famous of evolutionary algorithms. In a genetic algorithm, a population of individuals, they will survive in the environment according to their desirability. Those with superior capabilities will find greater the chance of marriage and reproduction. So, after a few generations, children with better performance are produced. In the genetic algorithm, each individual of the population is introduced into a chromosome. Chromosomes are more fully over several generations. In each generation, the chromosomes are evaluated, and they are enabled to survive and reproduce, according to its value. In terms of next generation genetic algorithm, takes place by the contact and mutation operators. Top of parents is elected on the basis of a fitness function [4]

Features and limitations of genetic algorithms

Genetic algorithms have properties that make it different and superior compared to other optimization algorithms, which refer to some of the most important ones, are as follows:

A) Genetic algorithms can make use of encrypted variables, ie chromosomes, instead of using the variable. As a result, it does not need to define the problem mathematically. So we can guess that, this algorithm can find answers to a wide range of issues.

B) Genetic algorithm applies the same time, a large number of parts to the space. This feature reduces the likelihood of being caught in the local optimum algorithm to a large extent.

C) Genetic algorithm does not guarantee convergence to local optimum, but most of the points have been optimized and converged accept.

D) This algorithm is used simply to replying the questions that a large number of variables.

E) The genetic algorithm is simple, and does not need the auxiliary information such as derivatives of the objective function. As a result, too complex for optimization of an objective function, batch or, systems that do not define specific material or direct actions by simulating the real system parameters are tested, very convenient.

As an alternative to genetic algorithm at the end of a call, a set of responses to provide optimal, this feature is important in multi-objective optimization problems.

However, their biggest constraint is how to write functional fitness (Fitness) which leads to the best solution to the problem. If this function fit, well and strong is not selected may be a solution to that problem is not found, or the wrong problem to be solved. [5]

In short, genetic algorithm is a programming technique, which uses genetic evolution as a problemsolving model. Entry is a problem that must be solved, and solutions are based on a template coding, the fitness function is called. Evaluates each candidate solution, most of which are randomly selected. They remain appropriate, and excluded the rest. The best people are together to mate (removable DNA elements) and go towards development. [6]

\section{GENETIC OPERATORS}

Genetic algorithms for solving problems (reproduction) follow three basic rules (operator) selection, integration and mutation (Figure 1).

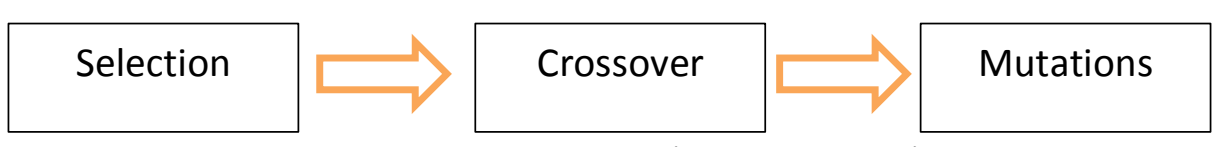

Diagram 1: genetic operators, writer 
Selection: The selection stage, a pair of chromosomes is selected to be combined to produce the next generation. In other words, the operator interface is between two generations and some members of the current generation are transmitted to the next generation. In electing the members of the compliance criteria and graceful them, but the selection process is random mode. Some roulette wheel selection procedures, are chosen sequence, select the Boltzmann constant mode, and so on.

Crossover: In nature, survival of generations, one of the most important factors and the only possible operator for this compilation is. Intercourse by switching genes between two chromosomes is done, and each of their chromosomes to offspring characteristics of next-generation transfer. This step is a process in which the older generation of chromosomes, mixed and combined, can bring up a new generation of chromosomes. The operator of genetic algorithms, function composition. Pairs who select as a parent are considered, in this field to exchange genes, and create new members. The most common methods are combined displacement binary, true displacement, combined single-point, twopoint combination, combining and so on. (Table 1)

Table 1: Some combine methods

\begin{tabular}{|l|l|l|l}
\hline Combination operator & $\begin{array}{l}\text { Parent chromosome } \\
\text { string 1 } \\
(\mathbf{0 0 1 0 0 0 1 1 1 )}\end{array}$ & $\begin{array}{l}\text { Parent chromosome string 2 } \\
(\mathbf{0 1 0 1 0 1 0 0 1 )}\end{array}$ & Child chromosome Strings \\
\hline one point Crossover & $\mathbf{0 0 1 0 0 / 0 1 1 1}$ & $\mathbf{0 1 0 1 0 / 1 0 0 1}$ & $\begin{array}{l}\mathbf{0 0 1 0 0 1 0 0 1} \\
\text { And } \\
\mathbf{0 1 0 1 0 0 1 1 1}\end{array}$ \\
\hline Two point Crossover & $\mathbf{0 0 1 0 / 0 0 1 / 1 1}$ & $\mathbf{0 1 0 1 / 0 1 0 / 0 1}$ & $\begin{array}{l}\mathbf{0 0 1 0 0 1 0 1 1} \\
\text { And } \\
\mathbf{0 1 0 1 0 0 1 0 1}\end{array}$ \\
\hline Multi-Point Crossover & & $\mathbf{0 1 0} / \mathbf{1 0} / \mathbf{1 0} / \mathbf{0 1}$ & $\begin{array}{l}\mathbf{0 0 1 1 0 0 1 0 1} \\
\text { And } \\
\mathbf{0 1 0 0 0 1 0 1 1}\end{array}$ \\
\hline
\end{tabular}

Mutation: a random change on people creates and produces new people in the population. The operator of a gene from a chromosome randomly (from a uniform distribution a bit random) selection and then alters gene content. If the gene is made of binary numbers, it's the inverse transform and if you belong to one or another element of its set value is set to replace the gene. Mutation operator to create a child uses only one parent; that would occur with small changes in the initial string. Mutation, makes searching the problem space intact, it is the main task of avoiding the convergence to local optimum mutation. [7] (Table 2)

Table 2: mutation

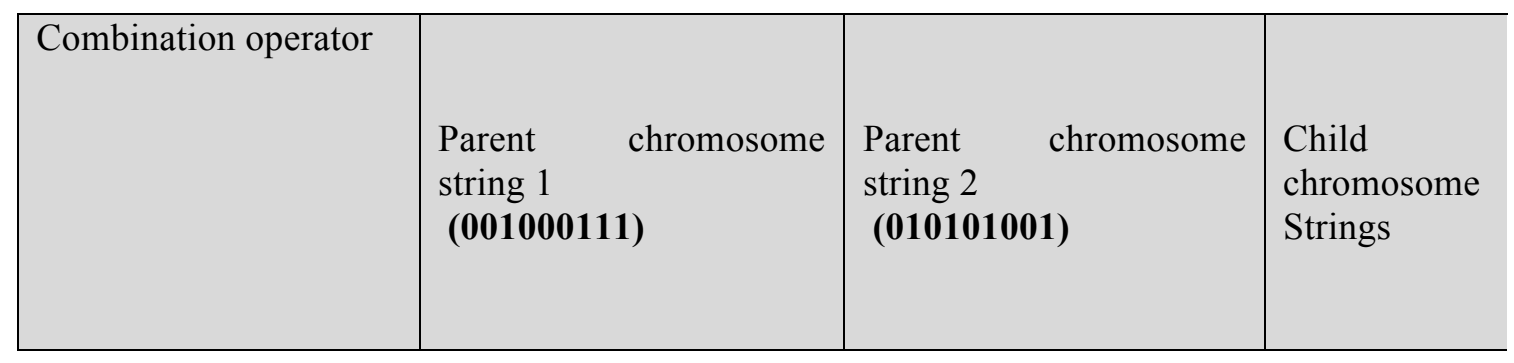




\begin{tabular}{|c|c|c|c|}
\hline mutation & 00100/0111 & 01010/1001 & $\begin{array}{l}001001111 \\
\text { Or } \\
010100001\end{array}$ \\
\hline
\end{tabular}

\section{STOPPING CRITERION}

Repeat operations until stop conditions continue to be met, and it is when a fixed number of generations, we dedicated budget or time runs out, a person (child produced) find the minimum (lowest), meet the criteria, fitting most of the children achieved better results not achieved or achieved one of the compounds mentioned above. [8] (Diagram 2)

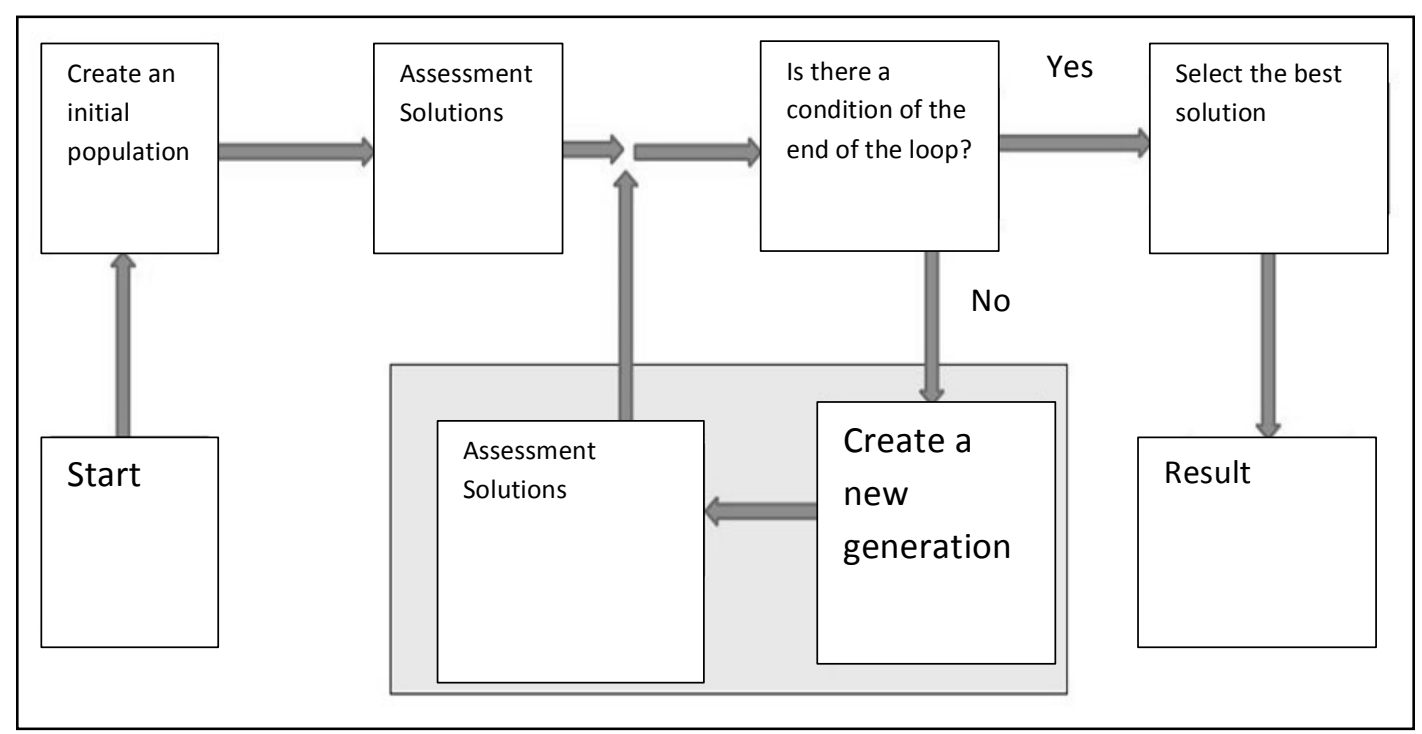

Diagram 2: The process of genetic algorithms performance, writer

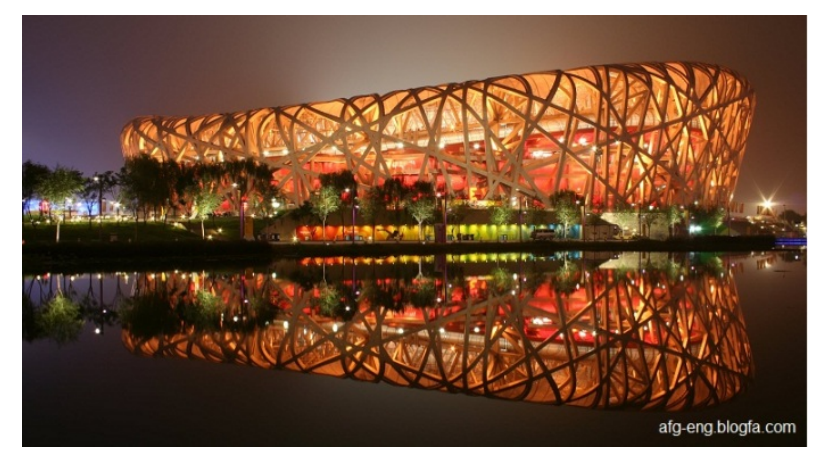

Figure 1: the Bird's Nest Stadium http://afg-eng.blogfa.com/post/52

Genetic computation, propose effective solutions for optimization problems and problem-solving issues through research, which applies to the population problem. Genetic algorithms, architectures are used in two ways: one as an optimizer tool, and the other as a production tool maker.

In the first method, the structural properties of genetic algorithms to problems such as structural performance, mechanical, thermal and lighting is concerned; that communication is more architectural and other building professionals. In the second method is under the new concept and fantastic, freedom in concept and form architecture. The design is based on the design parameters into the 
discussion. In the meantime, designers began the creation of free forms a complex with the building blocks is not easily understood by traditional methods. That's why; in this era of algorithms and programming codes that propels the science of architecture.

Unlike the conventional method of drawing volumes, modeling of the generation algorithm, is based on numbers, geometry and calculus based. Even if you begin high-volume design freedom, it must be connected volume initially specified parameters. The resulting routes, which are all linked and determined by changing parameters, the path has changed and volume calculations obtained is produced. [10]

\section{ALGORITHMIC DESIGN (PARAMETRIC- AMOUNT)}

If architecture is seen as a volume in space, the geometry and mathematics is dramatically visible in its design. Architectural history, every time the form has been associated with the geometry. Islamic architecture and decoration of buildings are examples, which show that architecture has a strong relationship with geometry and math. In recent times, due to the detachment of architecture and engineering fields this relationship weakened, but in the early 21 st century, more and more influential in the field of computer architecture, and helping to create new forms by the instrument, as well as the architecture of computer science Software found. By defining form as defined steps, called the socalled algorithms, new forms have gone back to the more scientific. This is a good thing, has led to a stronger relationship between the design and mathematical form. The combined fields of computational geometry (Computational Geometry) based on mathematical and computer programming, has led to the emergence of new intriguing topic generating algorithms (Generative Algorithm) geometry. The three-dimensional software, designers have the ability to have thought of almost any size can be drawn, but it is generating algorithms based design parameters has entered the field of design. In the meantime, designers to evaluate different volumes of Euclidean geometry began to design complex free-form curves and surfaces based on the release, which virtually are the building blocks of a process that is not easily understood by traditional methods to this. (Figure 2)

In general it can be said at this time, the power of algorithms and programming codes that propels the science of architecture. As a result, designers prefer to override the existing arena and beyond the usual volumes, with tools like algorithms generating fractal (Fractals), cellular automata (Cellular Automata), diagram (Voronoi), Linden Meyer System (L-System), triangulation (Triangulation), Premier formula (Super formula), and other specific algorithms. The horizon and the future move along with the diversity and creativity of the architect who dreams into reality are. [11]

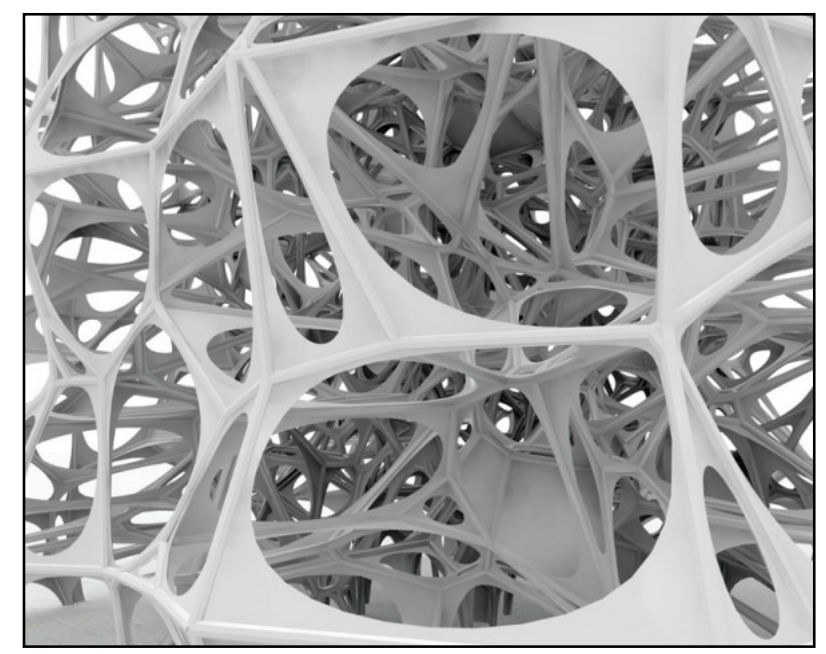

Figure 2: a diagram of Veronoi (divided space) http://matsysdesign.com/tag/voronoi 
Unlike the usual method of drawing volumes, with volume modeling algorithms based Productive numbers, geometry and calculations are based, even if the volume of design freedom begins, the volume must first be connected to the specified parameters. The result is that the routes are all linked and determined by changing parameters, the path has changed and volume calculations obtained is produced. (Figure 3)

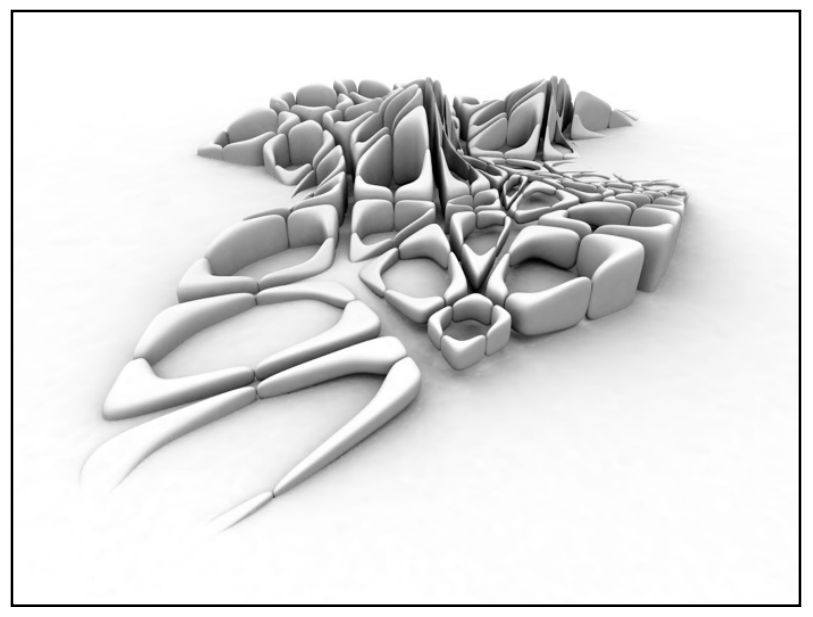

Figure 3: Urban Design Istanbul Turkey Kartal Pendik http://www.archello.com/en/project/kartal-pendik-masterplan/image-15

It is also necessary to note, that uses algorithmic methods to reduce the gross production volume differences between related disciplines such as architecture, engineering, energy, infrastructure, urban development and will be making arena. Software such as "Grasshopper" could well fill the gap between the design and construction as well. Science-based Code-low (CAD / CAM) are trying to make a connection between the building industry and architectural design, which is certainly "Grasshopper" is a tool that is able to make this connection as well. [11]

\section{CAPABILITIES OF PARAMETRIC ARCHITECTURE AND DESIGN PROCESS}

Patrick Schumacher, with a strong emphasis on the proposition that the convergence process approached those Parameter styles, such as a historical appreciation continues, noting: "Parameter the fundamental rethinking and constitute basic elements of architecture... Can not be the only one spectra or ethnic studied architecture, it should not even be described as experimental. Parameter, both in terms of architectural is solutions and the impact on the environment from the perspective of one of the largest and most important orientations and movements within the architectural discourse" .

Patrick Schumacher, stresses that the combination Parameter and digital design: "You can also use digital tools in traditional architecture, Parameter, the only direction is valid and reliable, the use of computer tools in new opportunities beyond the borders of all historical experience such as minimalism and develops high-tech. " [12]

In all, some of the capacities that provide parametric design or some of the architectural design process include:

possibility to create: Accounting and given the uncertain forms, is one of the most important problems that are among the preliminary design, final design and the architecture is, who are drawing the sketch because often in this sketch of free forms are drawn, are non renewal, because they do not follow the specified program. Efficient and diversified use of mathematical diagrams, the ability to provide, the distance between the initial and final design is reduced to a minimum. Easily applicable (Figure 4) 


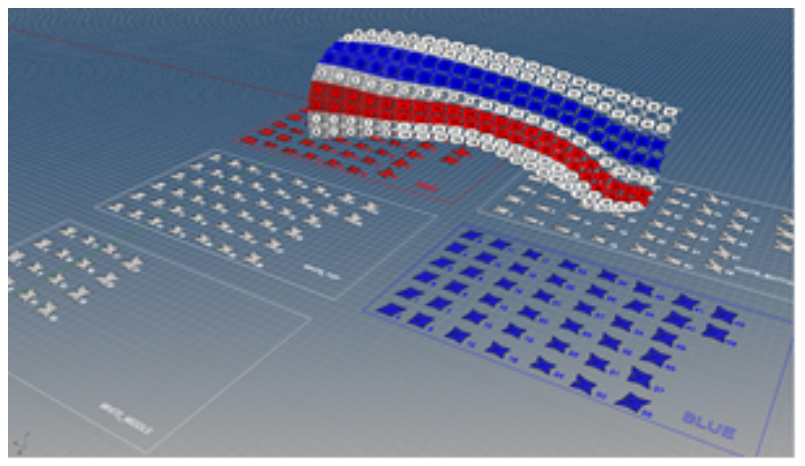

Figure 4: Presenting wide and numbered elements http://gracefulspoon.com/blog/2009/page/3

In conventional methods, designers are trying their feelings at the center of decision-making in the first place. So they invent new ideas to do outside of logical system of thought. This means that, architecture is always in the first stage of logical principles, and secondly, to be unaware of creativity and innovation. Another problem lies in the way of sensible ideas, is the possibility Reload manufacturer, meaning that, until the full embodiment, the architect can not come close to the ultimate goal, and if the project was conceived in the mind of the architect, making changes in it drastically reduced; in other words from irreversible, arises between logical thinking and artistic creativity.

Using mathematical ideas, the architects allows, always assured of the possibility of building their own creative ideas. Being repeatable mathematical graphs and the possibility of interference is constructive in the final product of options, it is easily solved, and this leads to more dynamism to the design process. On the one hand, the possibility of PCR amplification and sequencing, which is widely seen in mathematical diagrams, a rare approach, in order to create the possibility of building innovative and creative ideas to the architecture. (Figure 5)

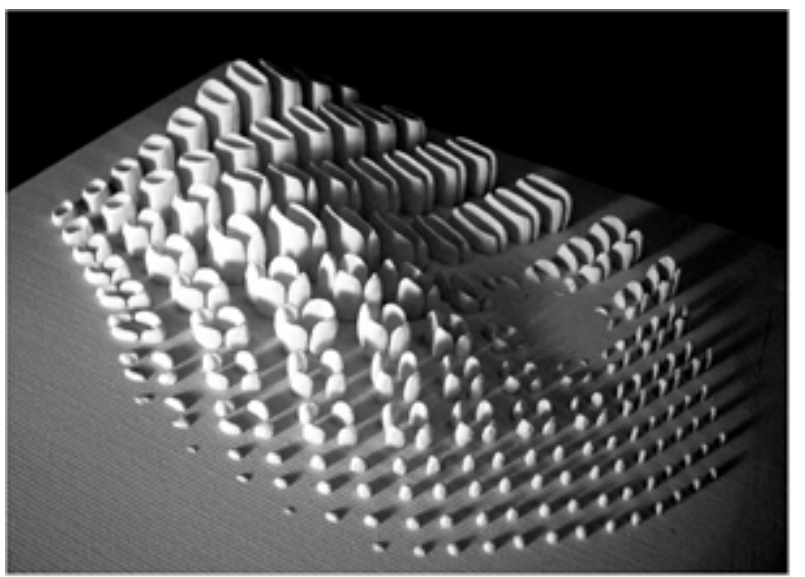

Figure 5: Urban Design Istanbul Turkey Kartal Pendik http://www.pinterest.com/aninekimer/urban-fabric

Compatibility form and structure: the boom of computer software, in architectural design, a new problem has developed in the field of architectural design. The computer software has caused to draw free curves, curves designed many are not manufactured in practice, or if they are built, there is no justification for making them economically. Such forms can be seen in many architectural competitions, which design is intended only visual proportions, and not a trace of concepts and mathematical proportions in them. Using defined mathematical principles, or general principles of modern mathematics could be a major step forward in improving this costly problem, be considered. Forms and mathematical proportions, is undeniably incompatible with the principles governing the 
design, construction and calculation of structures, inside. Lines, and curves load distribution, often with the help of famous mathematical functions are calculated. In cases where there are complexities basis, may be slightly hesitant to parse them into simpler formulas. Producer familiar with these concepts, instruments, is grappling with the problem that most architects. Using diagrams, and mathematical we proportion of the architecture that rare opportunity, at least subconsciously give reasonable answers to these questions. Forms, to help basic math concepts sketchy, often providing quality transmission structure is meaningfully guide. Architecture that takes advantage of this technique, in fact the management structure designed to coincide with the architectural design. [13] (Fig. 6)

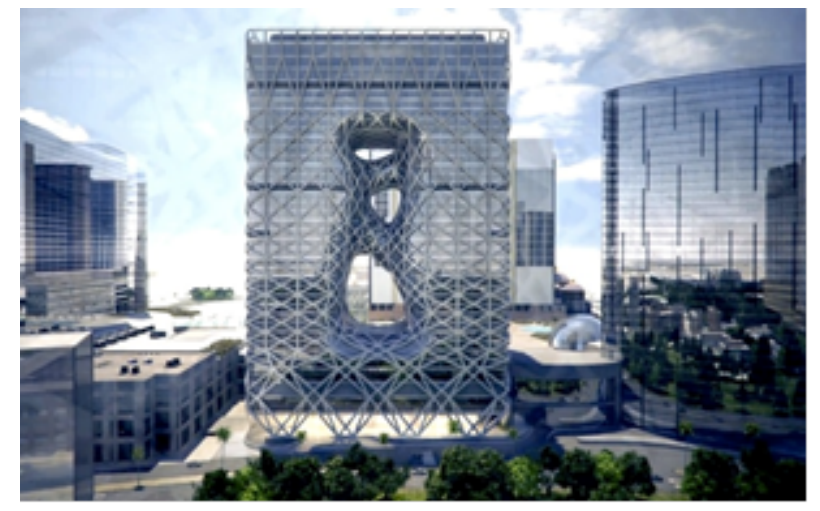

Figure 6: City of Dreams in Macau Tower Hotel

http://www.dezeen.com/2014/03/31/zaha-hadid-hotel-city-dreams-casino-resort-macau/

-Planning Optimization: Optimization refers to the process of improving anything. A designer creates new ideas and optimizes quality gives to the idea. [14]

In 1979, Judith Butler, Stefan M. Wild and Robert L.Phillips, provided the following definition of selectivity:

In many aspects of life, is not the best criterion for judgment, but it is progress towards best practice standards. Optimization, optimal point or points on your search, this definition has two parts: (1) progress and reform (2) optimal point. There is a distinct difference between process improvement and self-improvement concept.

Still considered important to converge on the study of optimized processes, (whether this is the best answer?), But the convergence towards the main issue is not the life more movements and only do things better than others is concerned. The main aim of the selectivity improvements to achieve optimum point is pervasive.

Genetic algorithm optimization considers every question, as a matter of evolution. This algorithm to find the optimal parameters, a set of possible values for them to randomly chosen as an initial population, then the process of evolution on this population, little by little they modify, which is the optimum value reach. Optimization theory how to obtain the best investigated. For this purpose, should be measured and how to recognize the difference between favorable and unfavorable. Optimization theory is to determine optimal locations and methods for finding it. [15]

Design optimization was introduced as a tool to achieve the best performance possible construction, highest reliability at the lowest cost, to the construction industry. Performance of buildings includes structural features, acoustics, lighting, energy and space in a building. For example, one of the main objectives of structural optimization is to minimize the total weight or materials cost reduction. With increasing global market demand for high-quality and modern buildings, the use of genetic algorithms to optimize one of the numerous techniques, is necessary. Especially for large structures, with thousands of hand calculations geometric element or structure can not meet the growing demand of 
the market. Therefore, the use of optimization techniques is inevitable. For example, in a project Aquatics Centre Olympic Games 2008 in Beijing, China, finding new ways to automatically select the size and Czech code 25000 pieces of steel were required to design them to possible project roof structure.

Most optimization problems, is caused by three main components: the first component of the objective function (objective function), which we're looking to increase or decrease the second component of the design of a set of variables, the value of the actual function (objective function) to affects and set a series of restrictions provides a possible design variables to achieve the eigenvalues of the third component.

For example, the structural performance of a panel, we determine what we want to increase or decrease (eg pressure on a specific area), then the variables that determine the geometry or materials forming the panel are, and then determine the limits of our which will minimize panel weight. [9]

\section{GA: NECESSITY OR ORIENTATION}

Increasing human needs and lifestyle today requires sophisticated application requirements and more creational forms which has been reinforced and the increasing complexity of manifestations and expressions of the form. The complexity and amount of information that is offered, one of the major problems is that the architecture is grappling with today. Today's problems can not be solved with traditional design methods. Also, limitations and bottlenecks of the problem, they understand and predict design performance has surpassed solutions. Taking into account the genetic algorithm as a tool to respond to the specific needs of the application architecture, not a mere experimental design, and the result is the idea of the necessity of the use of genetic algorithms and arise.

Since the design process is made up of many components, so they should not be overlooked, and thus should be focusing on symbols form. But since that form in architecture is a cultural tool, to visualize abstract concepts a lot depends on definition of evolution is not Darwinian. Architects must make clear that where the architecture is a part of nature, and analogies and metaphors in which they are placed, and where, the nature of inspiration.

Undoubtedly, abstract shapes, a conceptual world are completed in the future, so that it can be manifestations and expressions of a new form. Although the main challenges related to the use of the algorithm in real world situations, architecture, and of course the combination of complexity and limitations of evolutionary design problems with products form, to a design problem, and perhaps that is why the architecture of Genetic Algorithms use, or as a means of building and mechanical problems or to produce form.

In summary, despite the current difficulties, difficulties in encoding architecture, the processing algorithm as preparing the way the human mind to solve problems, be used in the design process. [9] Patrick Schumacher believes, has long been obvious that, the template is pervasive in contemporary architecture. Now, speaking of different versions of it, it must be repeated and efficiently. [16]

What has already described the importance and necessity of resorting to parametric architecture in modern architecture? Now, with the introduction of several architectural importance and necessity of the current trends that are transforming the mainstream architecture, the practical aspect is also taken into consideration. (Figures 7 to 11 ) 


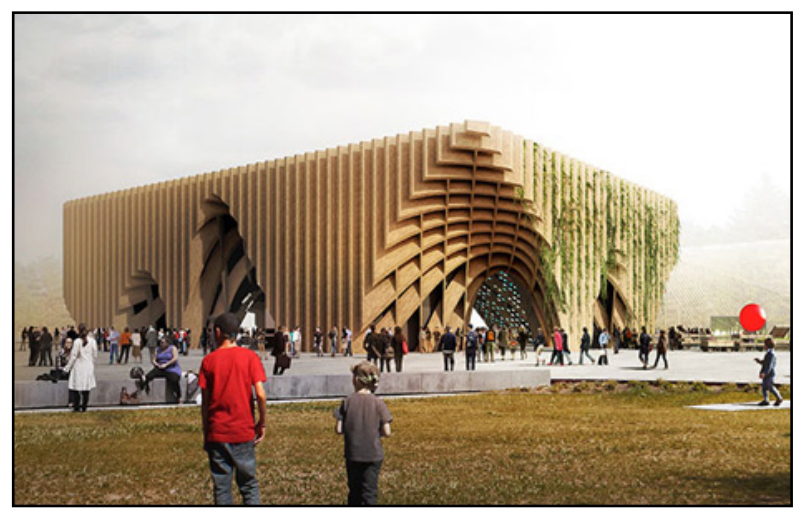

Figure 7: French Pavilion at Expo 2015 Italy

http://eater.com/archives/2014/04/30/french-architects-design-restaurant-that-grows-food-on-itswalls.php

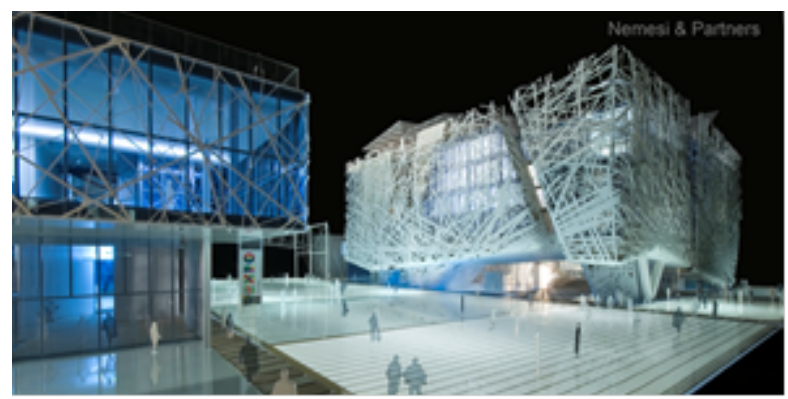

Figure 8: Qatar Pavilion at Expo 2015 Italy

http://www.e-architect.co.uk/milan/qatar-pavilion-expo-2015

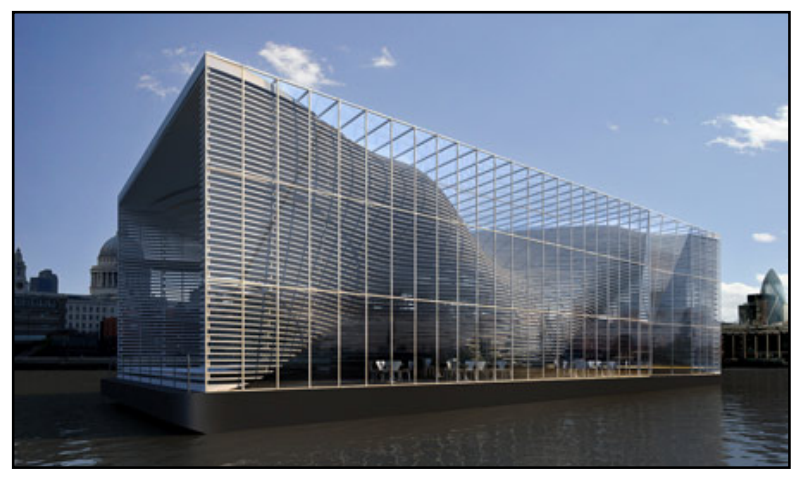

Figure 9: Italy Pavilion in Expo 2015 Italy

http://www.expocantiere.expo2015.org/il-progetto/padiglione-italia 


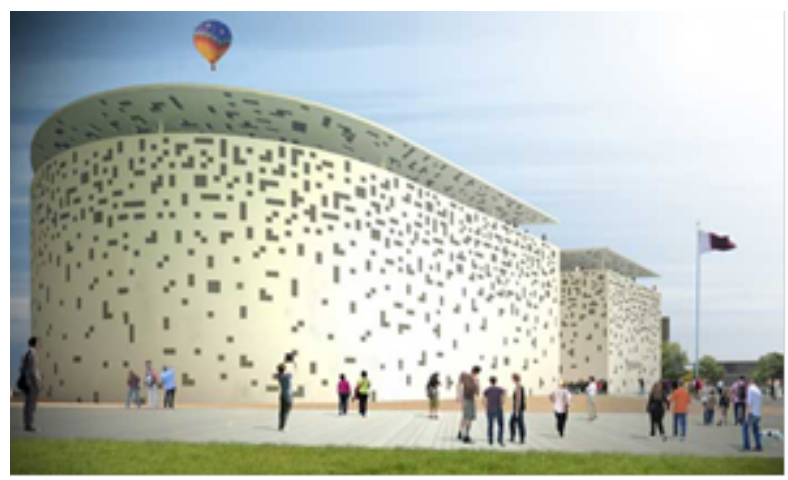

Figure 10: Gallery consistent architecture in London

http://www.metropolismag.com/Point-of-View/June-2012/Starting-a-Young-Practice

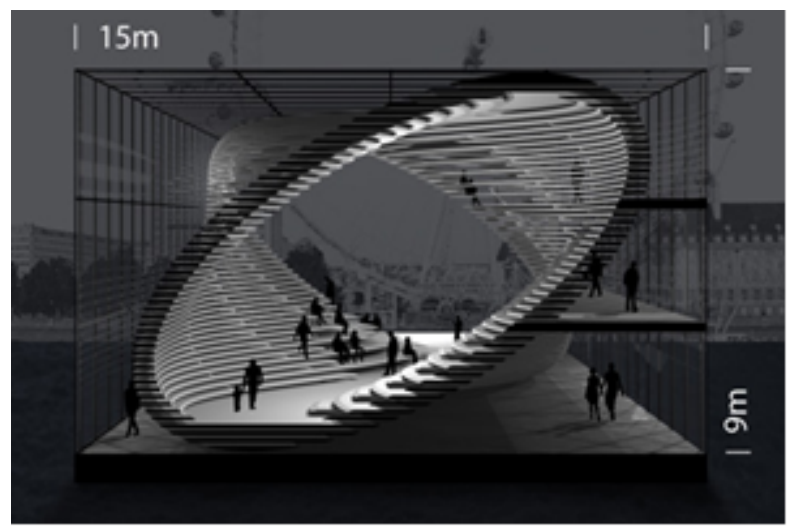

Figure 11: Gallery consistent architecture in London

http://www.dezeen.com/2009/01/21/adaptable-architecture-gallery/

\section{PROBLEM SOLVING PROCESS}

In the figure below (Figure 12) a fitting level based on variables A and B are shown.

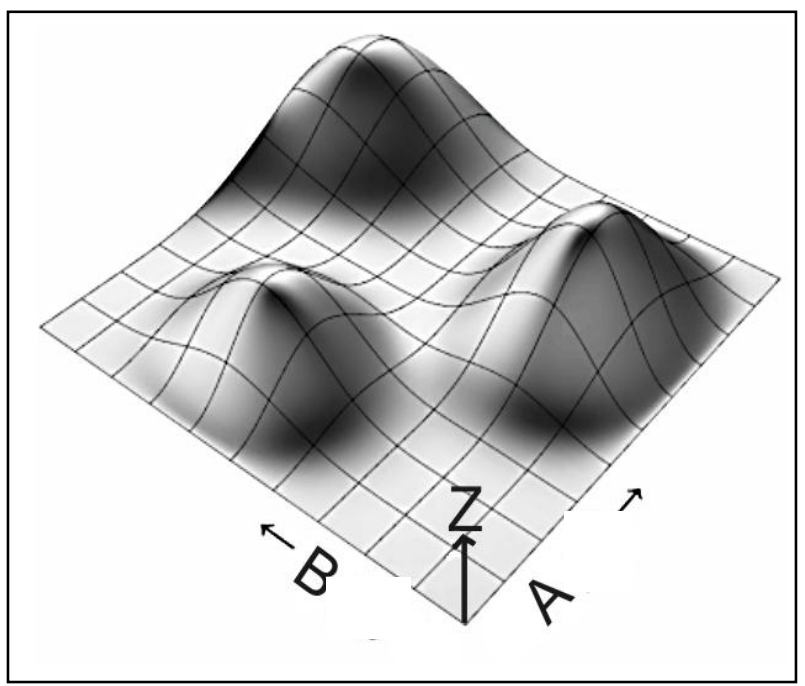

Figure 12: Level fitted (Z), based on the two genes (parameter) A and B http://www.grasshoppered.ir 
Two variables that level manufacturer, produce, $Z$ by a function. $Z=f(A, B)$, which in this case is unknown. If the function $f$ for all parts is known, by mathematical calculations Partial points can be awarded the maximum level, but because of unknown function, the manufacturer level, the objective is to find the maximum level (on the target) is. To solve the problem by evolutionary methods, to each of the parameters defined gene. A gene change may thus function better or worse, as a result of changes in gene a fitting point on the surface is high or low, but for any value A value B also can be modified, and re-fitting parts under the terms of the problem better or worse. For each combination of $A$ and $B$ is a value on the surface fitted, which operates the height $(Z)$ levels. The task of solving the problem of finding is highest point of the surface.

Sure, many of the issues will be defined not by genes but by multiple genes, and in this case, can not be used other than tangible example of the trend. A 12-gene model with a 12-dimensional abstract volume will be fitted in a space next 13 ! Because genes are one-dimensional and two-dimensional, tangible, followed by the limitations of evolutionary methods will be described. Moreover, it should be noted when the surface is fitted speaking; it may mean something much more complex than the images shown.

Starting problem solver algorithm, is since fitness level is unknown, a number of solutions to stochastic algorithm first on the scope of the problem of distribution. A genome is nothing more than the amounts specified for each of the genes (parameters). In the example above, a DNA can, for example, the value $\{0.2=\mathrm{A}$ and is $0.5=\mathrm{B}\}$. The algorithm then generates a random check their solutions, and the fitted value Calculates.

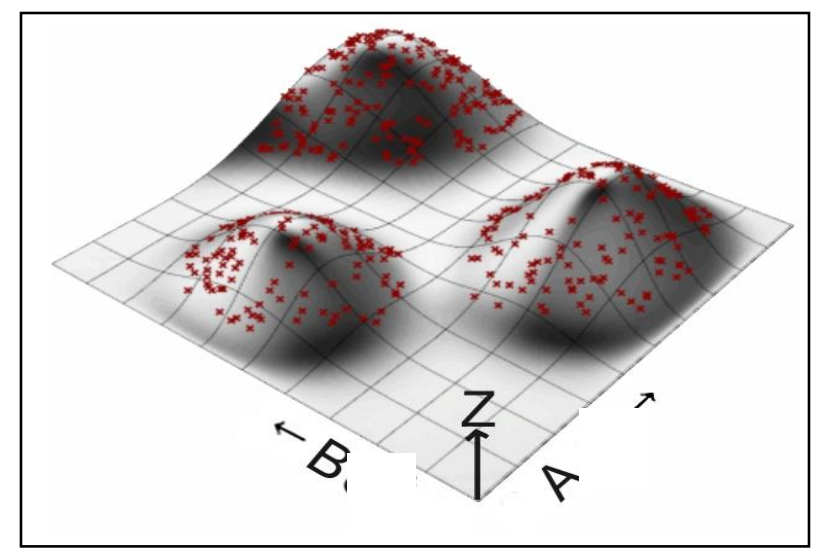

Figure 13 shows an example of a primary distribution evolutionary algorithm

After generating random algorithm solutions, review them, and the terms of the factors are fit. Figure 13 shows an example of a primary distribution evolutionary algorithm.

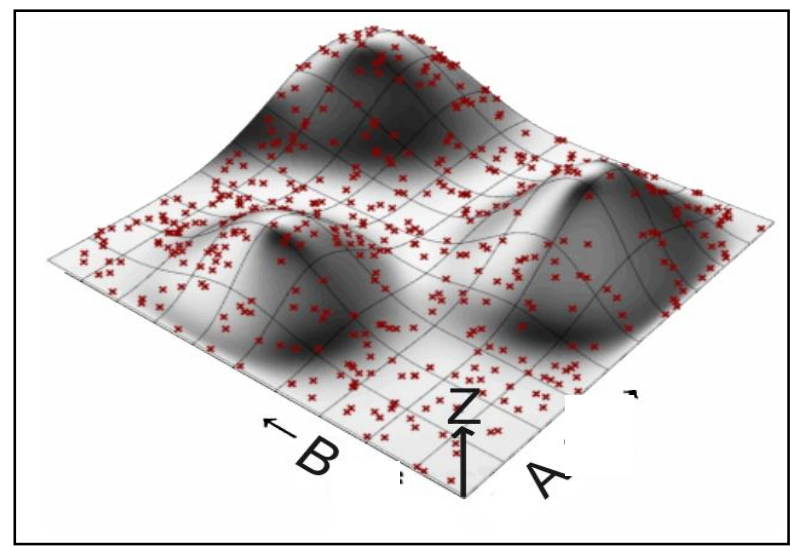


Figure 13 distribution points by the evolutionary algorithm

http://www.grasshoppered.ir

After the amount of each genome were obtained (the height of the example above) can be produced a list of best to worst solutions checked. Since that is the highest point, it is logical that genomes little more closely to the summit to smaller genomes, so you can remove the worst results obtained, and focus on better solutions.

Figure (14) better choices, according to the first generation of products, are shown.

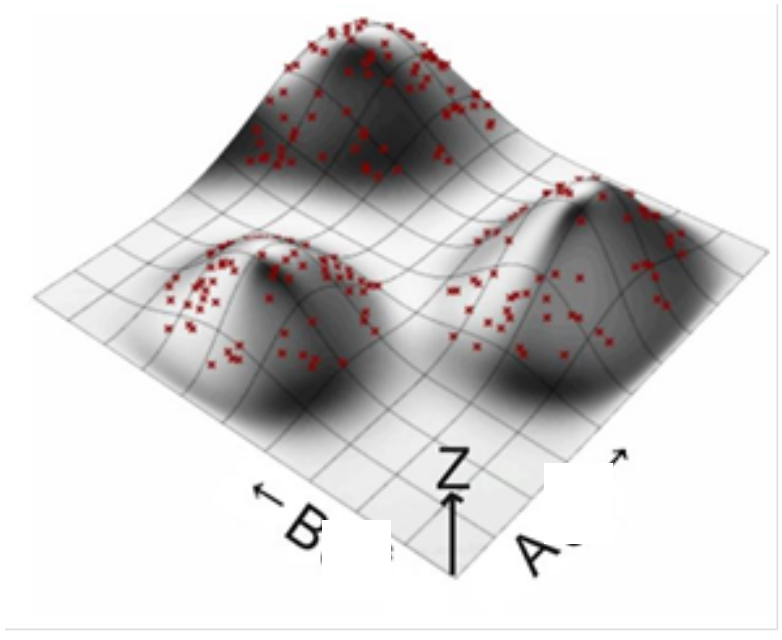

A figure (14) better choice, according to the first generation of products is shown.

Figure 14: Distribution Points by evolutionary algorithm

\section{http://www.grasshoppered.ir}

Leaving the app and use the solutions obtained in the first step of the algorithm is not useful enough because the first generation is randomly generated. The action that takes place at a later stage, the expansion of the genome to produce the next generation will be. When two favorites is together genome and produce a new generation generations will answer in the middle and as a result new solutions will be discussed. Figure (15) the operation is shown.

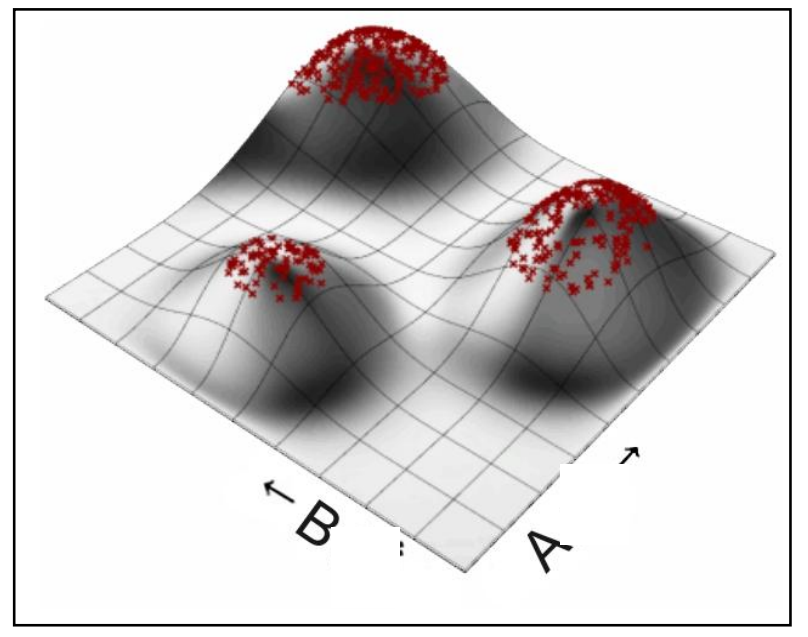

Figure 15: The points produced by combining two genome favorites

$$
\text { http://www.grasshoppered.ir }
$$


At this stage, a new generation is produced, which is not completely random, and begins to approach the maximum three points. Operations, continues to be a repeatable algorithm, eliminating the worst option and the best option to help generation to maximum points, which are practically lead to the solution of the problem. Figure (16) the process of evolutionary algorithm is shown. [17]

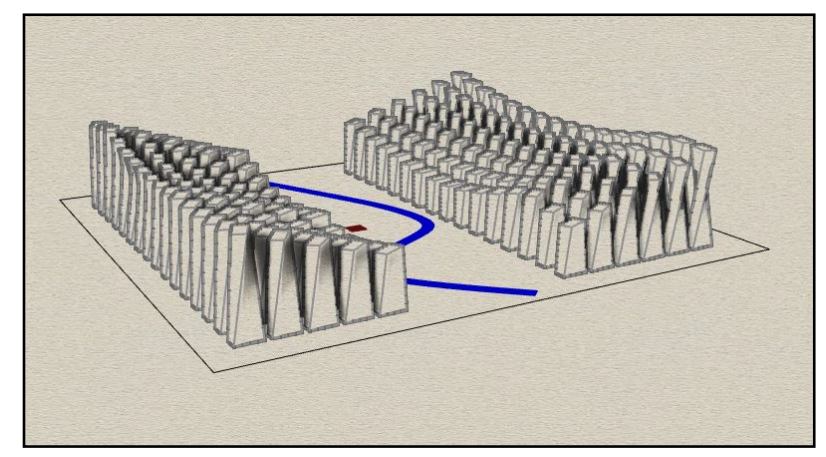

Figure 16: achieve maximum points as the solution of the problem by repeating the evolutionary algorithm

http://www.grasshoppered.ir

\section{ATTRACTOR POINT-ATTRACTOR LINE}

One of the most basic things that must be taken into account in the design of complex materials, according to precious metals is adjacent to the site, which is typical of the height, scattering, concentration etc., can be an effective set design.

In this section, in order to introduce more performance parametric software, outlines an example Rhino software and plug-in Grasshopper adjacent to a water route that passes through the site, and a historical element that will be discussed on the sidelines.

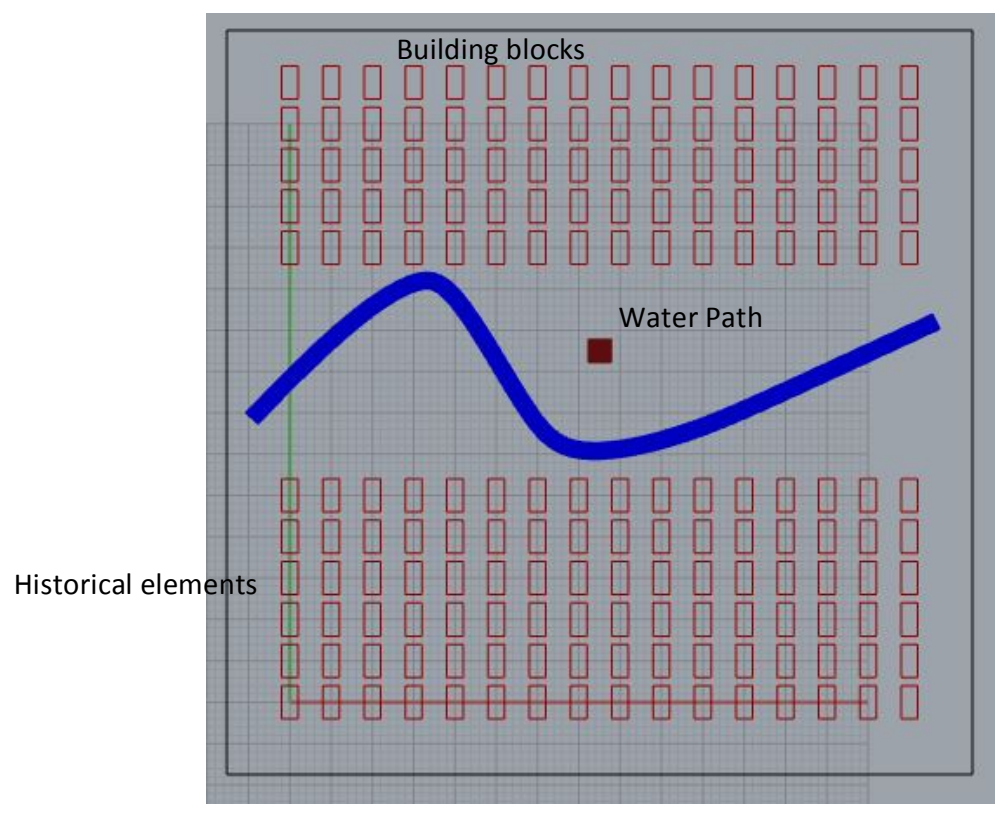

Figure 17: Site hypothetical

Following the issue under consideration, to obtain the optimum height of construction, so that, while proper height, due to the proximity of elements of historical value, depending on the value of the land take maximum advantage of the height. Also, turning buildings into is the important elements both for the aesthetics and the emphasis on them done. 
Factors affecting the design parameters, measuring the distance the water and the building with the site, the buildings reach a height of 6 to 18 meters around- in mind, according to Site-sight distance of the important elements affecting the neighboring valuable collection elements, and determine the distance and height of the rotation due to two factors.

What is obtained after the operations software, is as following images, which represents two things: First, do these operations without the help of algorithms and software requires a lot of time because they are perpendicular to obtain all grid points, to HYPOTHETICAL path defined, on the site, and its effect on the above example according to the network $16 \times 16$ and 96 point considered on the water, requires $96 \times 256$ operations which will require massive amounts of calculations, the(Figures 17 and 18)

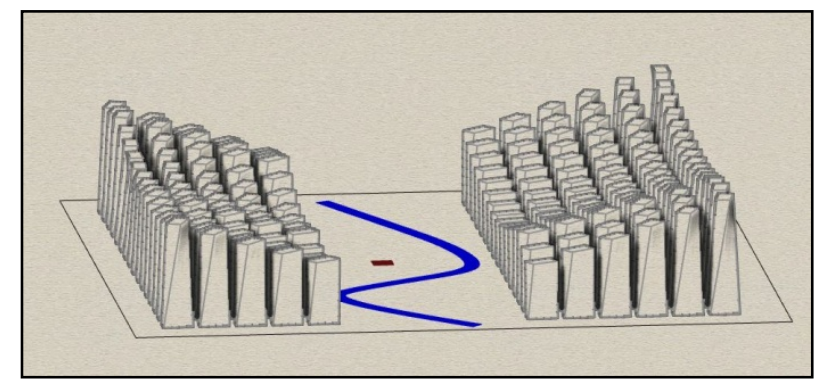

Figure 18: Elevation of Site

Figure 19: Elevation of Site

\section{CONCLUSION}

Genetic algorithms can be directed as a stochastic optimization method, which gradually moves toward the optimal point. About the characteristics of genetic algorithm, when compared with other optimization methods, we must say that algorithm that without having any knowledge of the problem and any limitation on the type of variable it is applicable to any problem, and have established efficiency in finding the optimal a whole. This method is the ability to solve complex problems of classical optimization methods or is not applicable or receivable is not reliable global optimum.

Due to parallel (non-linear) of these types of algorithms and consequently, a large space of solutions, has ability to search in several different problem areas.

One of the problems architects, information management and the increasing complexity involved in many contemporary projects. To deal with this problem recently builders have started using genetic algorithms in design. These algorithms both play a role in the design, production and optimization of novel forms. The first is the emergence of concepts and concept, and the latter as a means of solving the problems of structural, mechanical, thermal, lighting and more efficient.

Genetic algorithms are rapidly replacing traditional design process; and as soon as a need to have a more active role in the future of architecture.

Horizon ahead, show that: the architecture of the "slogans, form follows function", "less is more" and so on, in transition to another world, a world that no longer make any sense superior to another, and together the architecture of placement a set of parameters, in an overall composition, that each concept and form factor will have its own place. Spend a designer and architect of the state will become a strategist designer, one of the most important tasks will be to manage the various parameters.

\section{REFERENCES}

Abbas Kia, M., 1391, the meta-heuristic search algorithms, Tehran: IR PDF.

Alireza, Mahdi, 2007, "An Introduction to Genetic Algorithms and Applications", Tehran: Publishing Co., Bell thought 
Bayat, Mina, Pahelvanpour, R., 2013. "new challenge parametric architecture in Architecture and Urban Sustainability", International Conference of Civil Engineering, Architecture and sustainable development, Tabriz.

Classification", IEEE Int. Conference on Neural Networks, Pp. 1612-1616.

Fasoulaki, Eleftheria, 2007, "Genetic Algorithms in Architecture:a Necessity or a Trend?" Master of Science in Architecture, Computation Group Department of Architecture, Massachusetts Institute of Technology.

Gofee, William L. ,Ferrier, Gary D. , Rogers, John, 2000, "Global Optimization of Statistical Function With Simulated Annealing”, North-Holland: Journal of Econometrics , Pp. 65-99.

$\mathrm{http}: / /$ ahooramazdaa.mihanblog.com/post $/ 5$

http://grasshopper3d.ir

http://grasshopper3d.ir

http://rta-office.blogfa.com/post/1

http://www.etoood.com/NewsShow.aspx?nw=3648.

http://www.khalkhali.blogfa.com/cat-6.aspx

Kadivar, Arash, Sadeghi, MT, praised Karabakh, mercy, Mahmoud, Mehrak, 2010. "determine the kinetics of the hydrogenation process using an optimization approach", Journal of Chemical \& Petroleum Engineering, Volume 44, Number 1, pp. 82-73.

Khabbazi, Zubin, 2012. "paradigm algorithmic architecture", Mashhad: Ktabkdeh Kasra, p. 98.

Mitchel, Melanie, 1996," An Introduction to Genetic Algorithms", Massachusetts: MIT Press, Cambridge.

Rahimi, Rohollah, 2013. "Future Architecture and genetic algorithms", First National Conference on Renewable Energy and Clean, p. 6.

Tseng, L.Y. and Yang, S., 1997, "Genetic Algorithms for Clustering, Feature Selection and www.prozhe.com/ article Genetic Algorithm. Htm 